Supporting Information

\title{
A multi-functional droplet microfluidic platform for rapid immobilization of oligonucleotides on semiconductor quantum dots
}

\author{
Thu H. Nguyen ${ }^{1}$, Abootaleb Sedighi² ${ }^{2}$ Ulrich J. Krull ${ }^{2}$, Carolyn L. Ren ${ }^{1 *}$
}

1. Department of Mechanical and Mechatronics Engineering, University of Waterloo, 200 University Ave West, Waterloo, ON Canada

2. Department of Chemical and Physical Sciences, University of Toronto Mississauga, 3359 Mississauga Road, Mississauga, ON, Canada.

\section{S1. Detail of merging chamber design}

As discussed in the main text, the geometry of the merger has influenced the droplet fusion. The length of the chamber must be equal or larger than a merged droplet length $\left(L_{0}\right)$, identified by the number of merger droplets $(\mathrm{N}=2)$ multiplier of the input droplet length $\left(L_{d}\right)$. Herein, the merger is evaluated using the ratio (BRR) between the total bypass flow resistance $\left(R_{\text {Bybass }}\right)$ and the one of the mid-channel $\left(R_{c}\right)$ where the droplets fuses. Since that ratio is proportional to the ratio of oil flowing through each branch, BRR must be smaller than 1 to ensure that droplet will enter the central channel when it arrives at the chamber. In this work, the dimensionless of all parameters to design a merger chamber are shown as follows:

$$
\begin{gathered}
L_{p}^{*}=\frac{L_{p}}{W}=1 ; L_{s}^{*}=\frac{L_{s}}{W}=0.25 ; W_{p}^{*}=\frac{W_{p}}{W}=0.33 ; \\
L_{\text {relative }}^{*}=\frac{L_{d}}{L_{\text {chamber }}} ; \\
B R R=\frac{R_{\text {bypass }}}{R_{C}}=\frac{R\left(h, S_{p}, L_{p}\right)+\frac{1}{2}\left(h, W_{\text {bypass }}, L_{\text {chamber }}\right)}{R\left(h, W_{c}, L\right)}
\end{gathered}
$$

where $L_{p}$ is the length of each pillar, $L_{s}$ is the spacing between each pillar, $W_{p}$ is the width of each pillar, $L_{d}$ is the length of each droplet before merging, $W$ is the width of a main channel and $h$ is the height of an entire microfluidic network. 


\section{S2. A complex microfluidic circuit to an electric circuit analysis}

The functional performance of passive microfluidic designs mainly depends on channel dimensions and the precision of fabrication. The entire microfluidic network is well-connected, resulting in that any event happening in this network will change local and global hydrodynamic resistance. Thus, the microfluidic network, specifically a complex one, should be designed to be insensitive to multiple uncertainties, such as fabrication defects or local resistance changes, etc. The numerical simulation - Computational Fluid Dynamics (CFD) - can help to illustrate the fluid flow in detail and accurately; however, the high level of physical details as well as multiple parts integrated in one design will cause high computational cost.
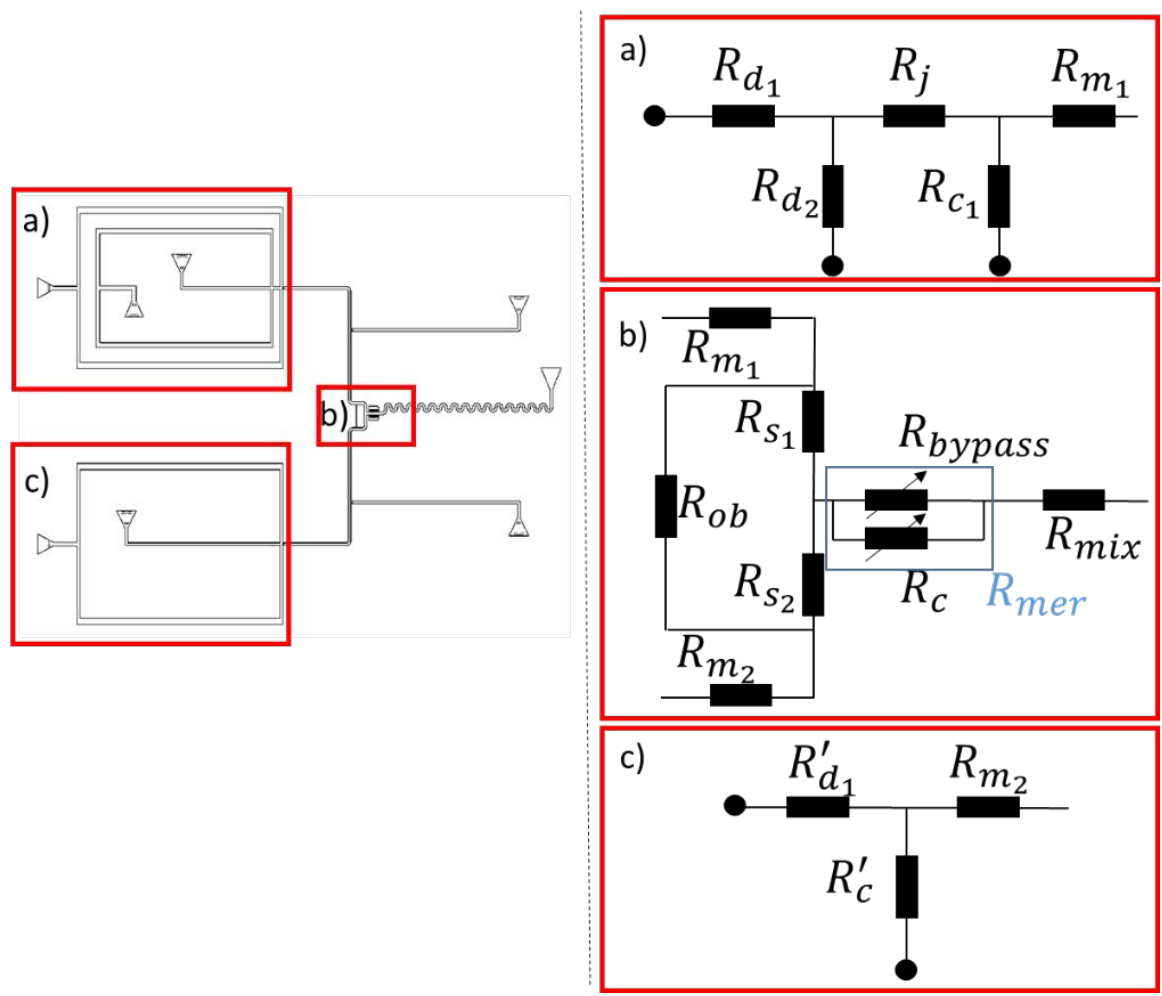

Figure S-1. Electric circuit analysis is used to simplify a complex droplet microfluidic system. a) upper part containing a double cross junction for co-encapsulating QDs and MBs; b) downstream part containing a droplet synchronizing component and a merging chamber; c) lower part containing a cross junction for generating droplets enclosing single stranded DNA oligonucleotides

Based on the analogous behavior of hydraulic and electric circuits with the associations of parameters (i.e, pressure to voltage, volumetric flow rate to current, hydraulic resistance to electric resistance, etc.), the applications of circuit methods are used to simplify a complex microfluidic design with multiple functions. The circuit analysis offers rapid adjustments of pressure-driven laminar flow in microchannels; therefore, the prognosis for the microfluidic design and the system's performance can be predicted in advance of fabrication. Particularly, this design includes 3 main parts, illustrated in Figure S- 
1. Part a) and c) represent the electric circuits that are used to simplify the encapsulation coupling with the droplet generator and another separate droplet generator, respectively. Details of design criteria for a double cross-junction for the encapsulation as well as the one for flow-focusing droplet generator are discussed in our previous work ${ }^{1}$ and in the work by Chen et al. ${ }^{2}$. Meanwhile, the alternative droplet merging component is illustrated in Part b). In detail, since the hydraulic resistances upstream are designed to be almost similar, as well as the flow conditions and the droplet generation frequencies of both generators are also maintained during the experiment, it will be valid to assume that $Q_{m_{1}}=Q_{m_{2}}$ is equivalent. Additionally, droplets are assumed to follow the flows of each branch. Therefore, there are 2 possible scenarios (Figure S-2).

1. Designing the two side branches to be symmetrical, $R_{S_{1}}=R_{S_{2}}$, leads to equivalent flow rates in the two side branches, $Q_{m_{1^{\prime}}}=Q_{m_{2^{\prime}}}$. Thus, the droplets from both sides will arrive at the junction at the same time and fuse into each other before they enter the chamber $\left(R_{m e r}\right)$.

(Figure S-2-1)

2. Designing two side branches are to be asymmetrical achieves alternating merging when the hydraulic resistance of one branch is slightly greater than that of the other, $R_{S_{2}} \leq R_{S_{1}}+R_{\text {drop }}$. At the beginning of a merging cycle, when both branches are filled with droplets, $R_{S_{2}}+1 R_{d r o p}$ $\leq R_{S_{1}}+2 R_{d r o p}$

- At the left note, $Q_{m_{1}}=Q_{m_{1}}-Q_{o b}(1)$

- At the right note, $Q_{m_{2}^{\prime}}=Q_{m_{2}}+Q_{o b}(2)$

Equations (1) \& (2) lead to $Q_{m_{1^{\prime}}}<Q_{m_{2^{\prime}}}$, resulting in the droplet from the right branch, which follows $Q_{m_{2}^{\prime}}$, moving faster than the ones from the left branch. Therefore, the right droplets will enter the chamber first and be partially trapped inside the pillar arrays (Figure S-2-2).

Subsequently, when a droplet leaves the right branch, the resistance of the right branch can be described as $R_{S_{2}}<R_{S_{1}}+R_{\text {drop }}$. The oil keeps draining from the left branch to the right branch, increasing $Q_{m_{2}^{\prime}}$ so that another droplet staying in the right branch tends to move forward to where it has higher flow rate. Meanwhile, the first droplet, staying in the left branch, also moves forward and temporarily blocks the junction, inducing built-up pressure. Continuously, another droplet from the right branch droplet comes and blocks the node right where the oil bridge and the main right channel meet. The flow in the oil bridge reverses its direction from right to left (Figure S-2-3). Now, the flow at the left branch increases through the addition of 
flow from the oil bridge (3), pushing remain droplets in the left branch to move forward until another coming droplet blocks a left node. A new merging cycle begins.

- At the left note, $Q_{m_{1}}=Q_{m_{1}}+Q_{o b}(3)$

- At the right note, $Q_{m_{2}^{\prime}}=Q_{m_{2}}-Q_{o b}(4)$

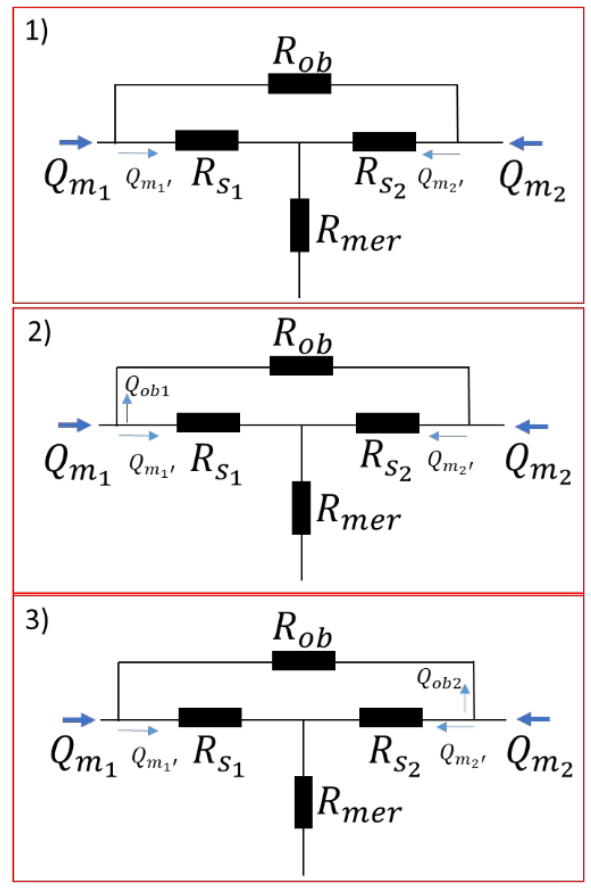

Scenario: Droplets fill the 2 asymmetrical side branches
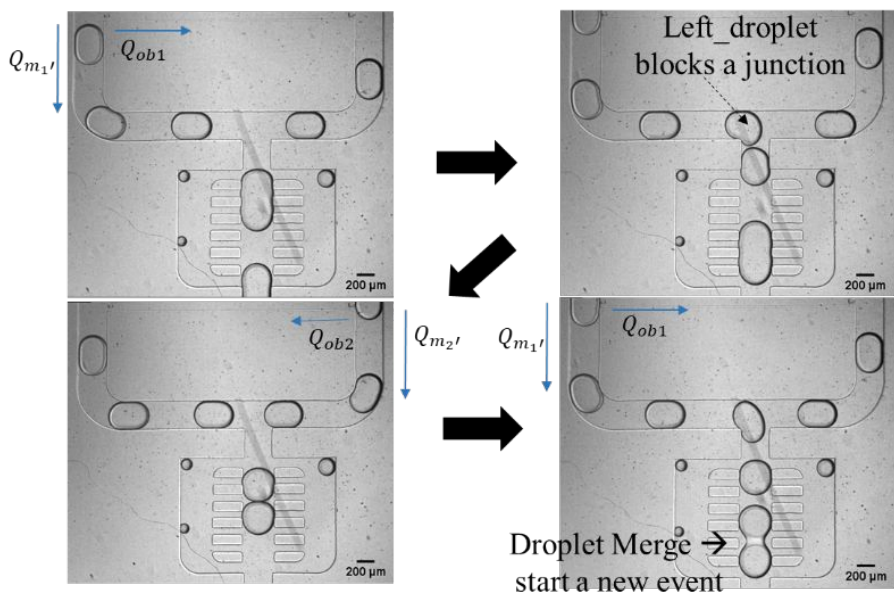

Figure S-2. The two scenarios show one-to-one droplet merging. (1) 2 side branches are symmetrical, (2) \& (3) 2 side branches are asymmetrical

The similarities between the physical parameters used in microfluidics and electronic parameters, which are useful in electric circuit analysis, are presented in Table S- 1 .

\begin{tabular}{|c|c|}
\hline Microfluidics & Electronics \\
\hline Fluid molecules & Electrons \\
\hline Flow of fluid & Flow of fluid \\
\hline $\begin{array}{l}\text { Volumetric flow rate } \\
\qquad Q\left[\frac{\mathrm{m}^{3}}{\mathrm{~s}}\right]\end{array}$ & $\begin{array}{l}\text { Electric current } \\
\qquad I[\mathrm{Amp}]\end{array}$ \\
\hline $\begin{array}{l}\text { Pressure drop } \\
\qquad \Delta P[\mathrm{~Pa}]\end{array}$ & $\begin{array}{l}\text { Voltage drop } \\
\qquad \Delta V[\text { Volt }]\end{array}$ \\
\hline $\begin{array}{l}\text { Hydraulic resistance } \\
R_{H}=\frac{12 \mu \mathrm{L}}{w h^{3}} \text { (for cases that } \mathrm{w} / \mathrm{h}<1 \text { and } \\
\text { for a rectangular channel) }\end{array}$ & $\begin{array}{l}\text { Electric resistance } \\
R=\frac{\rho . L}{A}\end{array}$ \\
\hline $\begin{array}{r}\text { Hagen-Poiseuille's law } \\
\qquad \Delta=Q \cdot R_{H}\end{array}$ & $\begin{array}{l}\text { Ohm's law } \\
\qquad \Delta V=I . R\end{array}$ \\
\hline
\end{tabular}




\begin{tabular}{|l|l|}
\hline $\begin{array}{l}\text { Equivalent fluidic resistor in series } \\
R_{e q}=R_{H_{1}}+R_{H_{2}}+\ldots\end{array}$ & $\begin{array}{l}\text { Equivalent electronic resistor in } \\
\text { series } \\
R_{e q}=R_{1}+R_{2}+\ldots .\end{array}$ \\
\hline $\begin{array}{l}\text { Equivalent fluidic resistor in } \\
\text { parallel } \\
1 / R_{e q}=\frac{1}{R_{H_{1}}}+\frac{1}{R_{H_{2}}}+\ldots\end{array}$ & $\begin{array}{l}\text { Equivalent electronic resistor in } \\
\text { parallel } 1 / R_{e q}=\frac{1}{R_{1}}+\frac{1}{R_{2}}+\ldots\end{array}$ \\
\hline Atmospheric pressure & Floating ground (GND) \\
\hline Conservation of mass & Kirchhoff's current law \\
\hline Conservation of energy & $\begin{array}{l}\text { Kirchhoff's votage law in a closed } \\
\text { path }\end{array}$ \\
\hline Pressure division & Voltage division \\
\hline Flow division & Current division \\
\hline
\end{tabular}

Table S-2. Physical similarities between microfluidics and electronics 


\section{S3. QD-DNA conjugation procedures}

The coating of glutathione on QDs:

The oleic acid-capped $\mathrm{CdSe} / \mathrm{ZnS}$ quantum dots were made water-soluble using a ligand exchange procedure with glutathione (GSH). Approximately, $0.4 \mathrm{~g} \mathrm{GSH}$ was dissolved in $1200 \mu \mathrm{L}$ of TMAH. Then, 1400 pmol QDs were dissolved in $4 \mathrm{ml}$ chloroform and gradually added to the GSH solution. Then, the solution was briefly vortexed and incubated overnight at room temperature. After incubation, the GSH-modified QDs were extracted to a top layer of $400 \mu \mathrm{L}$ borate buffer at $\mathrm{pH} 9.2$ containing $250 \mathrm{mM} \mathrm{NaCl}$. The organic layer was discarded and the aqueous layer was transferred to a $1.5 \mathrm{ml}$ centrifuge tube. $400 \mu \mathrm{L}$ ethanol was added to QD solution and the mixture was centrifuged at $8000 \mathrm{rpm}$ for $7 \mathrm{~min}$ to obtain a pellet of QDs. The procedure of buffer addition, ethanol precipitation and centrifugation were repeated two more times. Finally, GSH-QDs were re-dispersed in borate buffer $\mathrm{pH} 9.2$ (no $\mathrm{NaCl}$ ). The concentration of QDs was determined using UV-Vis absorption spectroscopy. An extinction coefficient value of $284,000 \mathrm{~cm}^{-1}$ was used to complete calculations for the absorption of the green-emitting QDs with the first exciton peak at $\sim 500 \mathrm{~nm}$.

Immobilization process:

Immobilization of DNA oligonucleotides on MB-loaded QDs occurs in two steps. The first step is the electrostatic-driven adsorption of oligonucleotides on the MB surfaces, and the second step is conjugation of adsorbed oligonucleotides to the QD surfaces through di-thiol (DTPA) coordination. The adsorption of oligonucleotides is driven by the electrostatic forces and creates a high concentration of oligonucleotides in the vicinity of QDs that substantially enhances the kinetics of the second step (The immobilization step). The immobilization step is a ligand exchange process, whereby the DTPA-oligonucleotides replace GSH groups on QD surfaces. 


\section{S4. The Red/Green ratio illustrating the nucleic detection}

The $\mathrm{R} / \mathrm{G}$ ratio, the case that $175 \mathrm{pmol}$ of oligonucleotide conjugating with QDs using this microfluidic platform, responses linearly in the presence of 50 pmol to 200 pmol Cy3-target DNA*.

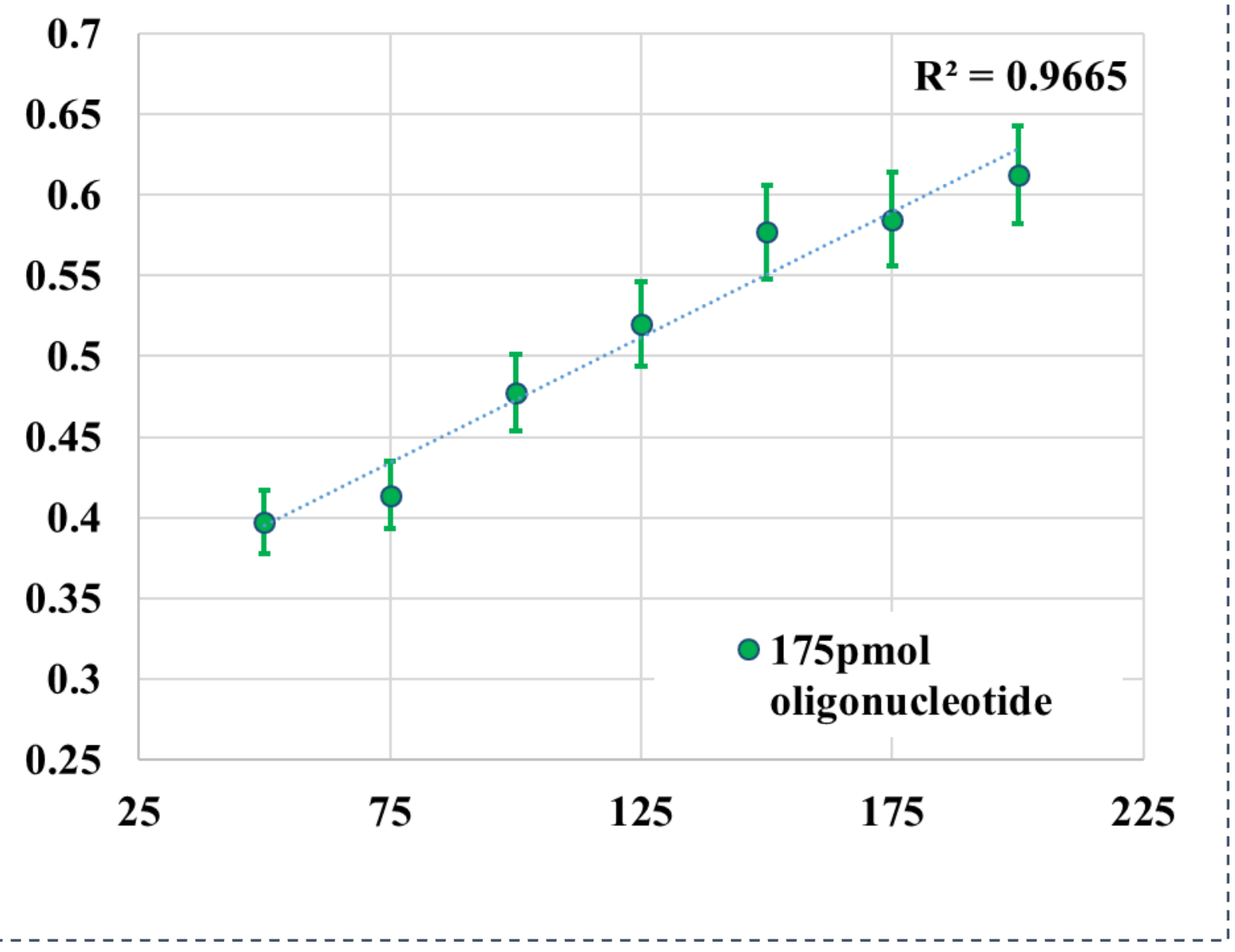

Figure S-3. R/G ratio illustrating the nucleic detection of the case that $175 \mathrm{pmol}$ ssDNA oligonucleotide is used to coated quantum dots where $y$-axis presents the $R / G$ ratio and $x$-axis presents the concentration of Cy3targeted DNA*

\section{References}

(1) Nguyen, T. H.; Chen, X.; Sedighi, A.; Krull, U. J.; Ren, C. L. A Droplet-Based Microfluidic Platform for Rapid Immobilization of Quantum Dots on Individual Magnetic Microbeads. Microfluid. Nanofluidics 2018, 22 (6), 1-11. https://doi.org/10.1007/s10404-018-2085-x.

(2) Chen, X.; Ren, C. L. Experimental Study on Droplet Generation in Flow Focusing Devices Considering a Stratified Flow with Viscosity Contrast. Chem. Eng. Sci. 2017, 163, 1-10. https://doi.org/10.1016/j.ces.2017.01.029. 